\title{
Some array polynomials over special monoid presentations
}

\author{
Ahmet Sinan Cevik ${ }^{1 *}$, Kinkar Chandra Das ${ }^{2}$, Yilmaz Simsek ${ }^{3}$ and Ismail Naci Cangul ${ }^{4}$
}

"Correspondence:

sinan.cevik@selcuk.edu.tr

'Department of Mathematics,

Faculty of Science, Selçuk University,

Campus, Konya, 42075, Turkey

Full list of author information is

available at the end of the article

\begin{abstract}
In a recent joint paper (Cevik et al. in Hacet. J. Math. Stat., acceptted), the authors have investigated the $p$-Cockcroft property (or, equivalently, efficiency) for a presentation, say $\mathcal{P}_{E}$, of the semi-direct product of a free abelian monoid rank two by a finite cyclic monoid. Moreover, they have presented sufficient conditions on a special case for $\mathcal{P}_{E}$ to be minimal whilst it is inefficient. In this paper, by considering these results, we first show that the presentations of the form $\mathcal{P}_{E}$ can actually be represented by characteristic polynomials. After that, some connections between representative characteristic polynomials and generating functions in terms of array polynomials over the presentation $\mathcal{P}_{E}$ will be pointed out. Through indicated connections, the existence of an equivalence among each generating function in itself is claimed studied in this paper.

MSC: 11B68; 11S40; 12D10; 20M05; 20M50; 26C05; 26C10
\end{abstract}

Keywords: minimality; characteristic polynomials; array polynomials

\section{Introduction and preliminaries}

In a recently published joint paper [1], the authors computed algebraic relations in terms of Ramanujan-Eisenstein series. Furthermore, although there are so many similar studies about figuring out the relationship between algebraic relations (or algebraic structures) and special generating functions ( $c f$., for instance, [2-5]; see also the references cited in each of them for other works) in the literature, we have not seen any such studies of relationship between (or monoid) presentations and generating functions. In fact, in terms of efficiency and inefficiency (while satisfying minimality) over group and monoid presentations, very important characterizations are given for related algebraic structures (see, for instance, [6-9]; see also the references cited in each of these earlier works). It is also well known that generating functions still attract much interest from all mathematicians and physicians (see, for instance, $[3,10]$ in addition to the above citations). Thus, it would be quite interesting for future studies to connect these two important areas and then search for possible properties about this linkage.

A similar connection exists between graphs and special functions since the number of vertex-colorings of a graph is given by a polynomial based on the number of used colors (see [11]). Based on this polynomial, one can define the chromatic number as the minimum number of colors such that the chromatic polynomial is positive. Recently, the paper [12] has come to our attention. It is a generalization on the chromatic polynomial of a graph subdivision, and basically the authors determine the chromatic number for a simple graph

(c) 2013 Cevik et al.: licensee Springer. This is an Open Access article distributed under the terms of the Creative Commons Attribution License (http://creativecommons.org/licenses/by/2.0), which permits unrestricted use, distribution, and reproduction in any medium, provided the original work is properly cited. 
and then present a generalized polynomial for the particular case of graph subdivision. In this reference, the main idea was to express some graph theoretical parameters in terms of special functions. In a similar manner within algebra, by considering a group or a monoid presentation $\mathcal{P}$, an approximation from algebra to analysis has been recently developed [13]. In this reference, the authors supposed that $\mathcal{P}$ satisfies the special algebraic properties either efficiency or inefficiency while it is minimal. (The reason for choosing efficiency or (minimal) inefficiency was to have an advantage to work on a minimal number of generators.) Then it was investigated whether some generating functions can be applied, and then studied what kind of new properties can be obtained by considering special generating functions over $\mathcal{P}$. Since the results in [12] imply a new studying area for graphs in the meaning of representation of parameters by generating functions, the results in [13] will also give an opportunity to make a new classification of infinite groups and monoids by using generating functions.

There has been also some interest in equivalence relations among generating functions (see, for instance, $[14,15]$ ). However in these studies, the authors were mainly interested in an equivalence relation that gives an opportunity to obtain generating functions one from another. In the literature, we have not seen any other paper that presents such a relation inside the related generating function. So, we believe that it is worth investigating whether there exist equivalence (or maybe congruence) relations among special generating functions depicted in here.

The ingredient of this paper should be thought of as not only computational but also theoretical. Hence it will be presented as three main parts including introduction. In Section 2, by reminding the results in [9], we will define characteristic polynomials related to the efficient and inefficient presentations, and then we will investigate the generating functions (in terms of array polynomials) over both these presentations and characteristic polynomials. In Section 3, we will provide some conclusions that will be studied as future projects.

\section{Generators for the semi-direct product of free abelian monoids rank two by finite cyclic monoids}

This main section will be given as three subsections under the names of Generating pictures (as Part I), Characteristic polynomials (as Part II) and Array polynomials (as Part III). In our study, we will consider a special presentation $\mathcal{P}_{E}$ (see (2) below). Since we will define characteristic polynomials and generating functions by considering the exponent sums of the generating pictures over $\mathcal{P}_{E}$, the first subsection is aimed at presenting these generating pictures and the related results about them.

\subsection{Part I: Generating pictures of $\mathcal{P}_{E}$}

It is strictly referred to [6-8] for fundamentals and properties of the algebraic subject used in this subsection. We further note that most of the material in here can also be found in [9].

We will mainly present the efficiency (equivalently, $p$-Cockcroft property for a prime $p$ ) for the semi-direct product of free abelian monoid $K_{2}$ having rank two by a finite cyclic monoid $A$ of order $k$. Hence, for $1 \leq l<k$ and $l, k \in \mathbb{Z}^{+}$, let $\mathcal{P}_{A}=\left[x ; x^{k}=x^{l}\right]$ be a presentation of $A$ and $\mathcal{P}_{K_{2}}=\left[y_{1}, y_{2} ; y_{1} y_{2}=y_{2} y_{1}\right]$ be a presentation of $K_{2}$. Suppose that $\psi$ is the endomorphism $\psi_{\mathcal{M}}$ of $K_{2}$, where $\mathcal{M}=\left[\begin{array}{ll}\alpha_{11} & \alpha_{12} \\ \alpha_{21} & \alpha_{22}\end{array}\right]$ such that the entries $\alpha_{i j}$ 's are the positive integers defined by $y_{1} \mapsto y_{1}{ }^{\alpha_{11}} y_{2}{ }^{\alpha_{12}}$ and $y_{2} \mapsto y_{1}{ }^{\alpha_{21}} y_{2}{ }^{\alpha_{22}}$. Hence the mapping $x \mapsto \psi_{x}$ 
$(x \in \mathbf{x})$ induces a well-defined monoid homomorphism $\theta: A \longrightarrow \operatorname{End}\left(K_{2}\right)$ if and only if $\mathcal{M}_{\left[x^{k}\right]}=\mathcal{M}_{\left[x^{l}\right]}$, or equivalently,

$$
\mathcal{M}^{k} \equiv \mathcal{M}^{l} \bmod d,
$$

where $d \mid(k-l)$ (see [9] for the details). Since there exists an ordering between the relators of $A$, that is, for $k, l \in \mathbb{Z}^{+}$, we have $1 \leq l<k$, it implies that to define an induced homomorphism $\theta: A \longrightarrow \operatorname{End}\left(K_{2}\right)$, that is, to define $K_{2} \rtimes_{\theta} A$, we must take congruence relation between $\mathcal{M}^{k}$ and $\mathcal{M}^{l}$ as given in (1) with the assumption $d \mid(k-l)$. By [9], the $k$ th and $l$ th powers of the matrices can be obtained by ordinary matrix multiplication. Thus, let us suppose that the $k$ th $\left(k \in \mathbb{Z}^{+}\right)$power of $\mathcal{M}$ is defined as

$$
\begin{aligned}
\mathcal{M}^{k} & =\left[\begin{array}{ll}
a_{k-1} & b_{k-1} \\
c_{k-1} & z_{k-1}
\end{array}\right]\left[\begin{array}{ll}
\alpha_{11} & \alpha_{12} \\
\alpha_{21} & \alpha_{22}
\end{array}\right] \\
& =\left[\begin{array}{ll}
a_{k-1} \alpha_{11}+b_{k-1} \alpha_{21} & a_{k-1} \alpha_{12}+b_{k-1} \alpha_{22} \\
c_{k-1} \alpha_{11}+z_{k-1} \alpha_{21} & c_{k-1} \alpha_{12}+z_{k-1} \alpha_{22}
\end{array}\right]=\left[\begin{array}{ll}
a_{k} & b_{k} \\
c_{k} & z_{k}
\end{array}\right],
\end{aligned}
$$

while, applying a similar idea, the $l$ th $\left(l \in \mathbb{Z}^{+}\right)$power of $\mathcal{M}$ is defined as $\mathcal{M}^{l}=\left[\begin{array}{l}a_{l} b_{l} \\ c_{l}\end{array}\right]$. Now suppose that (1) holds. Then the semi-direct product $E=K_{2} \rtimes_{\theta} A$ has a presentation

$$
\mathcal{P}_{E}=\left[y_{1}, y_{2}, x ; S, R, T_{y_{1} x}, T_{y_{2} x}\right],
$$

where $S: y_{1} y_{2}=y_{2} y_{1}, R: x^{k}=x^{l}, T_{y_{1} x}: y_{1} x=x y_{1}^{\alpha_{11}} y_{2}^{\alpha_{12}}$ and $T_{y_{2} x}: y_{2} x=x y_{1}^{\alpha_{21}} y_{2}^{\alpha_{22}}$, respectively. In the rest of this paper, we will assume that Equality (1) always holds when we talk about the semi-direct product $E$ of $K_{2}$ by $A$.

We know that the trivializer set (see [7]) of $\mathbf{X}_{\mathbf{E}}$ of $\mathcal{D}\left(\mathcal{P}_{E}\right)$ consists of the trivializer set $\mathbf{X}_{\mathbf{K}_{2}}$ of $\mathcal{D}\left(\mathcal{P}_{K_{2}}\right), \mathbf{X}_{\mathbf{A}}$ of $\mathcal{D}\left(\mathcal{P}_{A}\right)$ and the sets $\mathbf{C}_{1}, \mathbf{C}_{2}$ (see [8, Lemma 1.5]). In our case, by [16], $\mathbf{X}_{\mathbf{K}_{2}}$ is equal to the empty set since, for the relator $S$, we have $\iota\left(S_{+}\right) \neq \iota\left(S_{-}\right)$. Thus $\mathcal{P}_{K_{2}}$ is aspherical and so $p$-Cockcroft for any prime $p$. Nevertheless, the trivializer set $\mathbf{X}_{\mathbf{A}}$ of the Squier complex $\mathcal{D}\left(\mathcal{P}_{A}\right)$ can be found in [7, Lemma 4.4]. Finally, the subsets $\mathbf{C}_{1}$ and $\mathbf{C}_{2}$ contain the generating pictures $\mathbb{P}_{S, x}$ (which contains a non-spherical subpicture $\mathbb{B}_{S, x}$ as depicted in [7]), $\mathbb{P}_{R, y_{1}}$ and $\mathbb{P}_{R, y_{2}}$ of the trivializer set $\mathbf{X}_{\mathbf{E}}$. These pictures can be presented as in Figure 1(a) and (b).

For simplicity, let us define the sum of each entries of power matrices

$$
\left.\begin{array}{cccc}
a_{0}+a_{1}+\cdots+a_{k-1} & \text { as } \mathbf{a}_{k}, & a_{0}+a_{1}+\cdots+a_{l-1} & \text { as } \mathbf{a}_{l}, \\
b_{0}+b_{1}+\cdots+b_{k-1} & \text { as } \mathbf{b}_{k}, & b_{0}+b_{1}+\cdots+b_{l-1} & \text { as } \mathbf{b}_{l}, \\
c_{0}+c_{1}+\cdots+c_{k-1} & \text { as } \mathbf{c}_{k}, & c_{0}+c_{1}+\cdots+c_{l-1} & \text { as } \mathbf{c}_{l}, \\
z_{0}+z_{1}+\cdots+z_{k-1} & \text { as } \mathbf{z}_{k}, & z_{0}+z_{1}+\cdots+z_{l-1} & \text { as } \mathbf{z}_{l} .
\end{array}\right\}
$$

Suppose that the positive integer $d$, defined in (1), is equal to a prime $p$ such that $p \mid(k-l)$. Then, in [9], the following result has been recently obtained.

Proposition 1 ([9]) Let $p$ be a prime or 0. Then the presentation $\mathcal{P}_{E}$, as in (2), for the monoid $E=K_{2} \rtimes_{\theta} A$ is $p$-Cockcroft if and only if

(a) $\operatorname{det} \mathcal{M} \equiv 1 \bmod p$, 


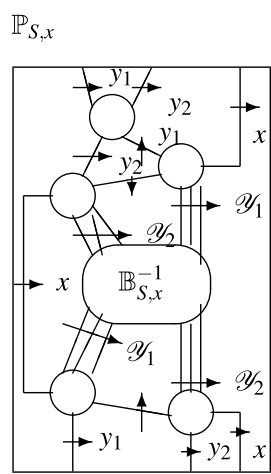

(a)
$\mathbb{P}_{R, y_{i}} \quad(i=1,2)$

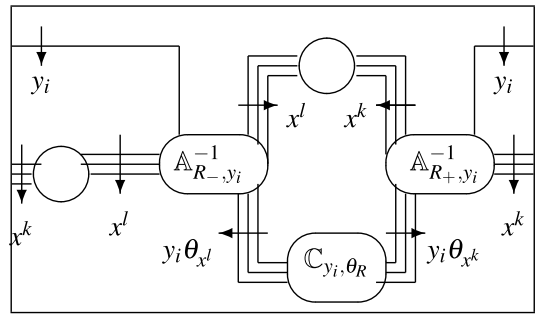

(b)

Figure 1 In the picture $\mathbb{P}_{S, x}$, for simplicity, relators $y_{1}^{\alpha} y_{2}^{\alpha}$ and $y_{1}^{\alpha} y_{2}^{\alpha} y_{22}^{\alpha}$ are labeled by $\mathcal{Y}_{1}$ and $\mathcal{Y}_{2}$, respectively.

(b)

$$
\begin{array}{llll}
\mathbf{a}_{k} \equiv \mathbf{z}_{l} & \bmod p, & \mathbf{b}_{k} \equiv \mathbf{c}_{l} & \bmod p \\
\mathbf{c}_{k} \equiv \mathbf{b}_{l} & \bmod p, & \mathbf{z}_{k} \equiv \mathbf{a}_{l} & \bmod p .
\end{array}
$$

According to the above proposition, let us take an efficient presentation

$$
\mathcal{P}_{E}=\left[y_{1}, y_{2}, x ; y_{1} y_{2}=y_{2} y_{1}, x^{2 p+1}=x, y_{1} x=x y_{1}^{\alpha_{11}} y_{2}^{\alpha_{12}}, y_{2} x=x y_{1}^{\alpha_{21}} y_{2}^{\alpha_{22}}\right]
$$

Suppose that $p$ is an odd prime. Then, in particular, $\mathcal{P}_{E}$ in (4) is not efficient if $\operatorname{det} \mathcal{M}$ is either equivalent to 0 or $p-1$ by modulo $p$. Therefore one of the consequences of Proposition 1 is the following.

Proposition 2 ([9]) The presentation $\mathcal{P}_{E}$ in (4) is minimal but inefficient if $p$ is an odd prime and

$$
\text { either }\left\{\begin{array} { l } 
{ \alpha _ { 1 1 } = p - 1 , } \\
{ \alpha _ { 1 2 } = \alpha _ { 2 1 } = 0 , } \\
{ \alpha _ { 2 2 } = 1 }
\end{array} \text { or } \left\{\begin{array}{l}
\alpha_{11}=1 \\
\alpha_{12}=\alpha_{21}=0 \\
\alpha_{22}=p-1
\end{array}\right.\right.
$$

\subsection{Part II: Characteristic polynomials over $\mathcal{P}_{E}$}

Let us reconsider the semi-direct product of $K_{2}$ by $A$ with a presentation $\mathcal{P}_{E}$ as defined in (2). Now it is well known that if one wants to define such a presentation $\mathcal{P}_{E}$, then it must satisfy condition (1). Therefore we certainly have $2 \times 2$-matrices $\mathcal{M}, \mathcal{M}^{k}$ and $\mathcal{M}^{l}$, and so we can consider the related characteristic polynomials of these matrices.

Let $\lambda_{1}$ and $\lambda_{2}$ be the only eigenvalues of the matrix $\mathcal{M}$. Since the entries of $\mathcal{M}$ are positive integers, $\lambda_{1}$ and $\lambda_{2}$ could be any numbers including complex ones. As a restriction, throughout all paper, we will assume that these eigenvalues are real. By using the basic fact of linear algebra, we then have the eigenvalues of $\mathcal{M}^{k}$ as $\lambda_{1}^{k}$ and $\lambda_{2}^{k}$ while the eigenvalues of $\mathcal{M}^{l}$ as $\lambda_{1}^{l}$ and $\lambda_{2}^{l}$. Thus, for a variable $v$, the characteristic polynomials over each of the 
matrices $\mathcal{M}, \mathcal{M}^{k}$ and $\mathcal{M}^{l}$ will be of the form

$$
\left.\begin{array}{l}
p_{k}^{l}(v)_{\lambda}=v^{2}-\left(\lambda_{1}+\lambda_{2}\right) v+\lambda_{1} \lambda_{2} \\
p_{k}^{l}(v)_{\lambda^{k}}=v^{2}-\left(\lambda_{1}^{k}+\lambda_{2}^{k}\right) v+\lambda_{1}^{k} \lambda_{2}^{k} \\
p_{k}^{l}(v)_{\lambda^{l}}=v^{2}-\left(\lambda_{1}^{l}+\lambda_{2}^{l}\right) v+\lambda_{1}^{l} \lambda_{2}^{l}
\end{array}\right\},
$$

respectively.

Now let us think of (5) as a piece of the system of characteristic polynomials that are obtained from any $2 \times 2$-matrix. In fact, there are an infinite number of polynomials of the type (5) since one can find an infinite number of matrices having positive integer entries that satisfy condition (1). On the other hand, by the definition of finite monogenic monoids and semigroups (see [17]), for a fixed value of $k$, one can choose the value $l$ from the set $\{1,2, \ldots, k-1\}$. It is clear that each of these systems in (5) will be constructed as a choice of $l$ in this set, and so we will have $k-1$ times different systems of characteristic polynomials as in (5) such that each of them contains an infinite number of polynomials. The following proposition will be based on this fact.

Proposition 3 Each characteristic polynomial obtained from $2 \times 2$ matrices, as in system (5), appears to be a congruence class.

Proof Before giving the proof, we note that this result can of course be adapted to $n \times n$ matrices, which will not be needed in here.

The normal form theorem [18], NFT for short, basically says that each congruence class contains a unique reduced word. It is well known in the branch of combinatorial group theory that the idea of this theorem is the main point when one tries to define a presentation for the related algebraic structure. Therefore, since each presentation $\mathcal{P}_{A}$ and $\mathcal{P}_{K}$ has been obtained by considering NFT, the presentation $\mathcal{P}_{E}$ in (2) must also satisfy NFT. (Although we reminded this theorem by considering only the words over free generators, the idea of NFT can actually be seen in $\mathbb{Q}$ as fractals or in matrix theory as echelon forms.)

For a fixed modulo $d$, let us think about the set, say $\Delta_{d}^{v}$, of all characteristic polynomials, written as in system (5), having the condition $\mathcal{M}^{k} \equiv \mathcal{M}^{l} \bmod d$, where $d \mid k-l$ and $l \in$ $\{1,2, \ldots, k-1\}$. We note that the cardinality $s\left(\Delta_{d}^{v}\right)=k-1$.

Nevertheless, for each different $1 \leq l \leq k-1$, since one can find matrices satisfying the condition in (1), the set $\Delta_{d}^{v}$ can be constructed as a union of $k-1$ times congruence classes, i.e., $l$-classes. Moreover, again by (1), for each $1 \leq l \leq k-1$, since we also have

$$
\lambda_{i}^{k} \equiv \lambda_{i}^{l} \equiv \lambda_{i} \quad \bmod d, \quad \text { where } 1 \leq i \leq 2,
$$

each of these $l$-classes contains the characteristic polynomials having eigenvalues $\lambda_{i}^{k}, \lambda_{i}^{l}$ and $\lambda_{i}$ (for $1 \leq i \leq 2$ ). On the other hand, by $(6), p_{k}^{l}(v)_{\lambda}$ can be taken as the simplest element (characteristic polynomial) having eigenvalues $\lambda_{1}$ and $\lambda_{2}$ in each $l$-class.

Thus, let us choose a power $l$ from the set $\{1,2, \ldots, k-1\}$ for a fixed $k$. Then the set $\Delta_{d}^{v}$ will be constructed for a suitable modulo $d$ according to our choice of $l$, which satisfies (1). Now, by (6), we have

$$
p_{k}^{l}(v)_{\lambda} \equiv p_{k}^{l}(v)_{\lambda^{k}} \equiv p_{k}^{l}(v)_{\lambda^{l}} \quad \bmod d
$$


in system (5). In other words, for a fixed $k$ and modulo $d$, there exists $\overline{p_{k}^{l}(v)_{\lambda}}=\overline{p_{k}^{l}(v)_{\lambda^{k}}}=$ $\overline{p_{k}^{l}(v)_{\lambda} l}$, where each class contains an infinite number of elements (polynomials). Therefore

$$
\overline{p_{k}^{l}(v)_{\lambda}}=\left\{p_{k}^{l}(v)_{\lambda}, p_{k}^{l}(v)_{\lambda^{k}}, p_{k}^{l}(v)_{\lambda^{l}}, \ldots\right\}
$$

where $1 \leq l \leq k-1$. As a summary, for a chosen $d$,

$$
\Delta_{d}^{v}=\left\{\overline{p_{k}^{l}(v)_{\lambda}}: k \in \mathbb{Z}^{+} \text {is fixed, } 1 \leq l \leq k-1, d \text { is fixed with } d \mid k-l\right\}
$$

such that $\bigcup_{1 \leq l \leq k-1} \overline{p_{k}^{l}(v)_{\lambda}}=\Delta_{d}^{v}$ and $\bigcap_{1 \leq l \leq k-1} \overline{p_{k}^{l}(v)_{\lambda}}=\emptyset$.

Hence the result.

As a next step of Proposition 3, the following result basically states that each semi-direct product presentation as in (2) which is obtained by condition (1) has a characteristic polynomial.

Theorem 1 The presentation $\mathcal{P}_{E}$ in (2) is represented by a unique (up to equivalence) characteristic polynomial defined as in system (5).

Proof By Proposition 3 and as a result of NFT, the simplest (unique reduced) characteristic polynomial in system (5) represents the congruence class of the related $l$-classes. From (1), we have $a_{k} \equiv a_{l} \bmod d, b_{k} \equiv b_{l} \bmod d, c_{k} \equiv c_{l} \bmod d$ and $z_{k} \equiv z_{l} \bmod d$, where $d \mid(k-l)$. Thus, by (6), we clearly obtain the equivalences

$$
\lambda_{1}^{k}+\lambda_{2}^{k} \equiv \lambda_{1}^{l}+\lambda_{2}^{l} \equiv \lambda_{1}+\lambda_{2} \quad \text { and } \quad \lambda_{1}^{k} \lambda_{2}^{k} \equiv \lambda_{1}^{l} \lambda_{2}^{l} \equiv \lambda_{1} \lambda_{2}
$$

by modulo $d$. Shortly, $\operatorname{tr}\left(\mathcal{M}^{k}\right) \equiv \operatorname{tr}\left(\mathcal{M}^{l}\right) \equiv \operatorname{tr}(\mathcal{M}) \bmod d$, where $\operatorname{tr}(\cdot)$ denotes the trace of these matrices, and $\operatorname{det} \mathcal{M}^{k} \equiv \operatorname{det} \mathcal{M}^{l} \equiv \operatorname{det} \mathcal{M} \bmod d$. This implies that each characteristic polynomial in system (5) is congruent to another by modulo $d$, and so the simplest polynomial

$$
v^{2}-\operatorname{tr}(\mathcal{M}) v+\operatorname{det} \mathcal{M}
$$

can be chosen as a representative characteristic polynomial for the presentation $\mathcal{P}_{E}$ in (2) since all these polynomials are in the same $l$-class. As a result, since $\mathcal{P}_{E}$ is obtained by using both unique reduced words (according to NFT) and the matrix $\mathcal{M}$ as the endomorphism $\psi_{\mathcal{M}}$, polynomial (8) represents this presentation uniquely as a characteristic polynomial.

As a consequence of Theorem 1 and Proposition 1 , we can give the following result which is a simpler version of Proposition 1 since the efficiency conditions can be expressed as a unique statement.

Theorem 2 For any prime $p$ or 0 , the presentation $\mathcal{P}_{E}$ in (2) is $p$-Cockcroft if and only if $\lambda_{1} \lambda_{2} \equiv 1 \bmod p$, where $\lambda_{1}<\lambda_{2}$ are the eigenvalues of the $2 \times 2$-matrix $\mathcal{M}$. 
Proof Now, by Theorem 1, it is known that $\mathcal{P}_{E}$ in (2) has a characteristic polynomial as in (8) with a base $2 \times 2$-matrix $\mathcal{M}$. Let $p$ be any prime or 0 , and let $\lambda_{1}<\lambda_{2}$ be the eigenvalues of $\mathcal{M}$. Thus, we clearly have $\operatorname{tr}(\mathcal{M})=\lambda_{1}+\lambda_{2}$ and $\operatorname{det}(\mathcal{M})=\lambda_{1} \lambda_{2}$. By Proposition 1 , since the sufficiency part is clear, let us show the necessity of the proof.

Assume that $\operatorname{det}(\mathcal{M}) \equiv 1 \bmod p$. Also, let us consider the power matrices $\mathcal{M}^{k}$ and $\mathcal{M}^{l}$ which certainly exist since $\mathcal{P}_{E}$ in (2) is a semi-direct product presentation. So, $\lambda_{1}^{k} \lambda_{2}^{k} \equiv 1$ $\bmod p$ and $\lambda_{1}^{l} \lambda_{2}^{l} \equiv 1 \bmod p$. In other words, by considering the sums presented in (3),

$$
\mathbf{a}_{k} \mathbf{z}_{k}-\mathbf{b}_{k} \mathbf{c}_{k} \equiv 1 \quad \bmod p \quad \Longrightarrow \quad \mathbf{a}_{k} \mathbf{z}_{k}=p t_{1}+1+\mathbf{b}_{k} \mathbf{c}_{k}
$$

and

$$
\mathbf{a}_{l} \mathbf{z}_{l}-\mathbf{b}_{l} \mathbf{c}_{l} \equiv 1 \quad \bmod p \quad \Longrightarrow \quad \mathbf{a}_{l} \mathbf{z}_{l}=p t_{2}+1+\mathbf{b}_{l} \mathbf{c}_{l},
$$

for some integers $t_{1}$ and $t_{2}$. Hence, by applying side by removing, we get

$$
\mathbf{a}_{k} \mathbf{z}_{k}-\mathbf{b}_{k} \mathbf{c}_{k} \equiv \mathbf{a}_{l} \mathbf{z}_{l}-\mathbf{b}_{l} \mathbf{c}_{l} \quad \bmod p
$$

which actually implies the truthfulness of the second part of (7).

On the other hand, by Theorem 1,

$$
\mathbf{a}_{k} \mathbf{z}_{k} \equiv \mathbf{a}_{l} \mathbf{z}_{l} \quad \bmod p
$$

since each characteristic polynomial in system (5) must be in the same $l$-class. From the above equivalence, we definitely get $\mathbf{a}_{k} \equiv \mathbf{z}_{l} \bmod p$ and $\mathbf{a}_{l} \equiv \mathbf{z}_{k} \bmod p$ since our assumption $\operatorname{det}(\mathcal{M}) \equiv 1 \bmod p$ does not permit another equivalence option. Furthermore, by keeping in our mind these last equivalences, if we consider (9) again, then we can get $\mathbf{b}_{k} \equiv \mathbf{c}_{l} \bmod p$ and $\mathbf{c}_{k} \equiv \mathbf{b}_{l} \bmod p$ since again the assumption on the determinant is enforced to not get another equivalence option.

Notice that the above processes show that this unique assumption on the determinant of $\mathcal{M}$ implies the remaining conditions of Proposition 1. Thus, as a next step of Proposition 1, we clearly get $\mathcal{P}_{E}$ is $p$-Cockcroft (equivalently, efficient) for any prime $p$ or 0 .

Example 1 By considering the matrix $\mathcal{M}$, one can give the following examples for Theorem 2:

(i) For an odd prime $p$, if we take $\alpha_{11}=1, \alpha_{12}=t\left(t \in \mathbb{Z}^{+}\right), \alpha_{21}=0$ and $\alpha_{22}=1$, then we get $\lambda_{1} \lambda_{2}=1$ while $\mathcal{M}^{p+1} \equiv \mathcal{M}^{1} \bmod p$. Hence, we get an efficient presentation. However, for even prime, we can still get a semi-direct product presentation for the same matrix entries since $\mathcal{M}^{3} \equiv \mathcal{M}^{1}$ mod 2. But the presentation will be inefficient.

(ii) For any prime $p$ and $t \in \mathbb{Z}^{+}$, again by taking

$$
\left[\begin{array}{ll}
1 & t \\
0 & 1
\end{array}\right] \text { or }\left[\begin{array}{ll}
1 & 0 \\
t & 1
\end{array}\right] \text {, }
$$

we can get $\mathcal{M}^{2 p+1} \equiv \mathcal{M}^{1} \bmod p$, and also $\lambda_{1} \lambda_{2}=1$. Hence we obtain an efficient presentation which can always be represented by a characteristic polynomial $(v-1)^{2}$ for both cases. 
(iii) Similarly, for any prime $p$, by choosing ei ther $\alpha_{11}=p t+1\left(t \in \mathbb{Z}^{+}\right), \alpha_{12}=0$, $\alpha_{21}=0$ and $\alpha_{22}=1$ or $\alpha_{11}=1, \alpha_{21}=0, \alpha_{12}=0$ and $\alpha_{22}=p t+1\left(t \in \mathbb{Z}^{+}\right)$, we can still obtain efficient presentations with the equivalence $\mathcal{M}^{2 p+1} \equiv \mathcal{M}^{1} \bmod p$. Further, the representative characteristic polynomial for the related presentation is of the form $v^{2}-(p t+2) v+p t+1$.

(iv) The above examples can be extended for $\mathcal{M}^{n p+1} \equiv \mathcal{M}^{1} \bmod p$, where $n \in \mathbb{Z}^{+}$.

As another consequence of Theorem 1, we obtain the following result by considering Proposition 2.

Theorem 3 For an odd prime $p$, the presentation $\mathcal{P}_{E}$ in (4) is minimal but inefficient if $\mathcal{M}$ is a diagonal matrix and the representative characteristic polynomial is of the form $v^{2}-p v+(p-1)$ which has a strict ordering $1<(p-1)$ between roots.

Proof Let $\mathcal{M}$ be a diagonal matrix. For an odd prime $p$, let $v^{2}-p v+(p-1)$ be the representative characteristic polynomial for $\mathcal{P}_{E}$. Clearly, the roots 1 and $p-1$ will be the eigenvalues $\lambda_{1}$ and $\lambda_{2}$ of $\mathcal{M}$. Then, by (8), we have $\operatorname{tr}(\mathcal{M})=p$ and $\operatorname{det}(\mathcal{M})=p-1$. By Proposition 2, this determinant value implies that $\mathcal{P}_{E}$ is inefficient. Notice that since $\mathcal{P}_{E}$ in $(4)$ is a semi-direct product presentation, $\mathcal{M}^{2 p+1} \equiv \mathcal{M} \bmod p$ must be held. Therefore we have $\lambda_{1}^{2 p+1} \equiv \lambda_{1}$ $\bmod p$. So, by Proposition 3, the polynomial with roots $\lambda_{1}^{2 p+1}$ and $\lambda_{2}$ is in the same congruence class as the simplest characteristic polynomial $v^{2}-p v+(p-1)$. In addition, by the assumption, the entries of $\mathcal{M}$ should be either $\alpha_{11}=1, \alpha_{22}=p-1$ or vice versa $\alpha_{12}=0=\alpha_{21}$. Then, again by Proposition $2, \mathcal{P}_{E}$ is minimal, as required.

Example 2 Let $p=3$ and $\mathcal{M}=\left[\begin{array}{ll}1 & 0 \\ 0 & 2\end{array}\right]$ with determinant 2. Thus $\mathcal{M}^{7} \equiv \mathcal{M} \bmod 3$ holds, and the relators of the semi-direct product presentation, as in (4), will be $y_{1} y_{2}=y_{2} y_{1}, x^{7}=x$, $y_{1} x=x y_{1}$ and $y_{2} x=x y_{2}^{2}$. By Theorem 3 , the simplest (unique) characteristic polynomial $v^{2}-3 v+2$ represents this inefficient but minimal presentation. A similar example can also be obtained by considering the matrix $\left[\begin{array}{ll}2 & 0 \\ 0 & 1\end{array}\right]$.

Conjecture 1 Let us consider a $2 \times 2$-matrix $\mathcal{M}$ with eigenvalues $\lambda_{1}$ and $\lambda_{2}$, respectively. Then, by considering $\operatorname{tr}(\mathcal{M})=\lambda_{1}+\lambda_{2}$ and $\operatorname{det}(\mathcal{M})=\lambda_{1} \lambda_{2}$, one can investigate whether the minimality conditions of Theorem 3 can be expressed as a unique statement

$$
\operatorname{gcd}(\operatorname{tr}(\mathcal{M}), \operatorname{det}(\mathcal{M}))=1
$$

\subsection{Part III: Array polynomials over $\mathcal{P}_{E}$}

In this section, we are mainly interested in the generating functions (in terms of array polynomials) related to the presentations defined in (2) and (4). In [13, 19], by considering the generating pictures in two different group and monoid extensions, the authors have investigated the related generating functions over the presentations of them. However, in a different manner, here we will investigate the array polynomials (as generating functions) in the meaning of characteristic polynomials obtained in the previous section. In other words, by taking into account Proposition 3 and Theorems 1, 2 and 3, we will reach our aim using semi-direct products of monoids studied in this paper.

As noted in [13, Remark 1.1], if a monoid presentation satisfies efficiency or inefficiency (while it is minimal), then it always contains a minimal number of generators. Working 
with a minimal number of elements gives a great opportunity to define related generating functions over this presentation. This will be one of the key points in our results.

Our first result of this section is related to the connection of the monoid presentation in (2) with array polynomials. In fact, array polynomials $S_{a}^{n}(x)$ are defined by means of the generating function

$$
\frac{\left(e^{t}-1\right)^{a} e^{t x}}{x !}=\sum_{n=0}^{\infty} S_{a}^{n}(x) \frac{t^{n}}{n !}
$$

(cf. [20-22]). According to the same references, array polynomials can also be defined as follows:

$$
S_{a}^{n}(x)=\frac{1}{a !} \sum_{j=0}^{a}(-1)^{a-j}\left(\begin{array}{l}
a \\
j
\end{array}\right)(x+j)^{n} .
$$

Since the coefficients of array polynomials are integers, they find a very large application area, especially in system control ( $c f$. [23]). In fact, these integer coefficients give us an opportunity to use the polynomials in our case. We should note that there also exist some other polynomials, namely Dickson, Bell, Abel, Mittag-Leffler etc. having integer coefficients which will not be handled in this paper. Now, by using (10) and keeping in our mind that the coefficients of array polynomials are integers, we clearly have

$$
S_{a}^{n}(b)= \begin{cases}b^{n} ; & a=0 \\ b ; & a=0 \text { and } n=1, \\ 1 ; & a=n \text { or } n=a=0 .\end{cases}
$$

Therefore we have the following theorem.

Theorem 4 The presentation $\mathcal{P}_{E}$ defined in (2) has a set of generating functions $p_{1}(x)=$ $\left[S_{0}^{k}(x)\right]-\left[S_{0}^{l}(x)\right]$ and

$$
p_{2}\left(y_{i}\right)=S_{0}^{1}(x)+\left[S_{0}^{\alpha_{i m}}\left(y_{1}\right)\right]+\left[S_{0}^{\alpha_{i m}}\left(y_{2}\right)\right]+\frac{1}{S_{0}^{1}(x)},
$$

where $S_{a}^{n}(x)$ and $S_{a}^{n}\left(y_{i}\right)$ are defined as in (11), $\alpha_{i m}$ 's are the entries of $\mathcal{M}$ and $1 \leq i, m \leq 2$.

Proof Let us consider the presentation $\mathcal{P}_{E}$ in (2). We recall that for $\mathcal{P}_{E}$ to be a semi-direct product presentation of the free abelian monoid $K$ rank two by cyclic monoid $A$ of order $k$, by (1), the power matrices $\mathcal{M}^{k}$ and $\mathcal{M}^{l}\left(1 \leq l<k\right.$ and $\left.l, k \in \mathbb{Z}^{+}\right)$must be congruent to each other by modulo $d$, where $d \mid k-l$. By the meaning of endomorphism, this congruence actually comes from the existence of the relator

$$
x^{k}=x^{l}
$$

where $x$ is the unique generator of $A$. In this relator, by replacing $x$ by an array polynomial $S_{0}^{1}(x)$ and also considering the status modulo $d$, we can get $p_{1}(x)$ as a generating function. Basically, the function $p_{1}(x)$ must exist in all such sets that contain generating functions of the presentation $\mathcal{P}_{E}$ in (2). 
Now, let us give our attention to investigating the existence of generating functions of the form $p_{2}\left(y_{i}\right)$. Actually, the existence of these functions is again based on the existence of the equality in (1). Because the relators

$$
y_{1} x=x y_{1}^{\alpha_{11}} y_{2}^{\alpha_{12}} \text { and } y_{2} x=x y_{1}^{\alpha_{21}} y_{2}^{\alpha_{22}}
$$

have been obtained by the meaning of endomorphism on the base matrix $\mathcal{M}$, it is clearly seen that while the first row of $\mathcal{M}$ gives the above first composite relator, the second row gives the second one. Thus, by replacing $y_{1}$ by the array polynomial $S_{0}^{1}\left(y_{1}\right)$ and $y_{2}$ by the array polynomial $S_{0}^{1}\left(y_{2}\right)$ into the above relators, we get the required functions. Notice that although in monoids we are not allowed to apply the inverse element $x^{-1}$, by the meaning of functions, we can use this inverse element as a fractal of the form $\frac{1}{S_{0}^{1}(x)}$.

Notice also that in the light of the above material, for the relator $y_{1} y_{2}=y_{2} y_{1}$, the generating function will only be the zero function.

Nevertheless, by considering Theorem 1 and Equation (8), we can also get the following result.

Theorem 5 The presentation $\mathcal{P}_{E}$ defined in (2) has a single generating function

$$
p(v)=S_{0}^{2}(v)-\left(\lambda_{1}+\lambda_{2}\right) S_{0}^{1}(v)+\left(\lambda_{1} \lambda_{2}\right) S_{n}^{n}(v),
$$

where $v$ represents three-ordered variables $\left(x, y_{1}, y_{2}\right)$ and $\lambda_{1}, \lambda_{2}$ are the eigenvalues of $\mathcal{M}$.

Proof Since, by Theorem 1, the presentation $\mathcal{P}_{E}$ can be represented by a unique characteristic polynomial as in (8), the function obtained from this polynomial should be the unique generating function. However, since this unique function must represent the whole presentation, it should be constructed by all generators of $\mathcal{P}_{E}$. In other words, the required function should be defined as multi-variable $\left(x, y_{1}, y_{2}\right)$. Hence, by replacing variables by array polynomials as previously, we can get the generating function as given in (12).

Conjecture 2 By taking into account Theorems 1, 4 and 5, one can investigate whether there exists an equivalence between the generating functions that represent the presentation $\mathcal{P}_{E}$ in (2) as indicated in Figure 2.

By considering Proposition 1, Theorem 2 and Theorem 4, respectively, we further obtain the following corollary.

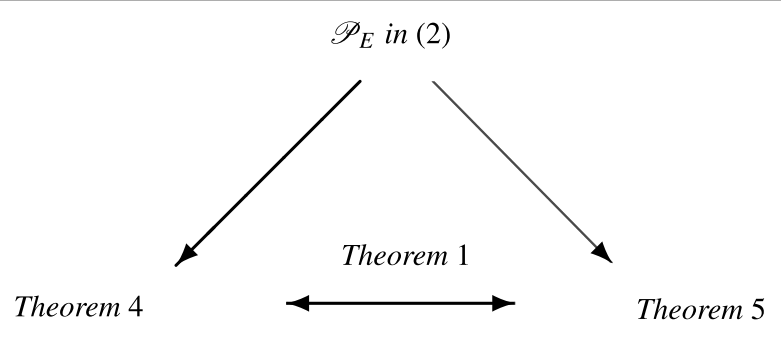

Figure 2 Possible equivalence between generating functions. 
Theorem 6 Suppose $\mathcal{P}_{E}$ in (2) is an efficient presentation. Then it accepts

$$
p_{3}(x)=\left[\lambda_{1} \lambda_{2}-1\right] S_{0}^{1}(x)
$$

as a generating function including the functions defined in Theorem 4 , where $S_{a}^{n}(x)$ is defined as in (11) and $\lambda_{1}, \lambda_{2}$ are the eigenvalues of $\mathcal{M}$.

Proof By Theorem 4, we have the generating functions $p_{1}(x)$ and $p_{2}\left(y_{i}\right)(1 \leq i \leq 2)$. However, since $\mathcal{P}_{E}$ is given as an efficient presentation, we also need to add a function related to its efficiency. At this stage, we can think about Theorem 2 which basically says that $\mathcal{P}_{E}$ is efficient if $\lambda_{1} \lambda_{2}-1 \equiv 0 \bmod p$. Moreover, this condition is directly related to the generator $x$ in $\mathcal{P}_{E}$ ( $c f$. [9, Theorem 2.4]). Therefore this last generating function should be of the form $\left[\lambda_{1} \lambda_{2}-1\right] S_{0}^{1}(x)$, as required.

The following corollary is a consequence of Theorem 5 which states that the expression of Theorem 6 can also be given as a single condition.

Corollary 1 Suppose $\mathcal{P}_{E}$ in (2) is an efficient presentation. Then it accepts $p(v)=S_{0}^{2}(v)-$ $\left(\lambda_{1}+\lambda_{2}\right) S_{0}^{1}(v)+1$ as a generating function, where $v$ represents three-ordered variables $\left(x, y_{1}, y_{2}\right)$ and $\lambda_{1}, \lambda_{2}$ are the eigenvalues of $\mathcal{M}$.

The sketch of proof Since the efficiency of a presentation implies $\lambda_{1} \lambda_{2} \equiv 1 \bmod p$ for any prime $p$ or 0 , we can take $\lambda_{1} \lambda_{2}=1$ in (12) because, by NFT, the simplest (reduced) form represents all other generating functions $p(v)$. Thus, depending on the constants, the function $p(v)$ in the result can be thought to be in the congruence class of all such functions.

Remark 1 From Corollary 1 and by considering Theorem 1, we can easily deduce that the set of generating functions for an efficient presentation can be presented as a single element. This actually shows the importance of efficiency during the study of generating functions.

In Proposition 2 and also in Theorem 3, we expressed the minimality (while satisfying inefficiency) of the presentation $\mathcal{P}_{E}$ in (4) in two different versions, respectively. As a next step of Theorem 6 and as a consequence of Theorem 4, we will deal with the array polynomials which are obtained from a minimal but inefficient presentation. The following lemma will be needed in the proof of our next result.

Lemma 1 There always exists $(p-1)^{2 p} \equiv 1 \bmod p$ for any prime $p$.

Proof We first note that the lemma is clear for $p=2$. So, let us assume that $p$ is an odd prime.

Suppose that $(p-1)^{2 p} \equiv 0 \bmod p$. So, by the meaning of congruence, we must have $(p-1)^{2 p}=p t$ for a $t \in \mathbb{Z}^{+}$. In fact, the left-hand side of this equality can be written as a binomial sum

$$
(p-1)^{2 p}=p^{2 p}-2 p^{2 p}+\left(2 p^{2}-p\right) \cdot p^{2(p-1)}-\cdots+1 .
$$


In this sum, each term except the last one, which is 1 , is congruent to 0 by modulo $p$ since each of them contains $p$ as a factor. Thus, $(p-1)^{2 p}$ cannot be congruent to 0 by modulo $p$ unless we add -1 to both sides of this congruence. Therefore $(p-1)^{2 p} \equiv 1 \bmod p$, as required.

Theorem 7 For an odd prime $p$, the minimal but inefficient presentation

$$
\mathcal{P}_{E}=\left[y_{1}, y_{2}, x ; y_{1} y_{2}=y_{2} y_{1}, x^{2 p+1}=x, y_{1} x=x y_{1}^{p-1}, y_{2} x=x y_{2}\right]
$$

has a set of generating functions

$$
\begin{array}{ll}
p_{1}(x)=\left[S_{0}^{2 p+1}(x)\right]-\left[S_{0}^{1}(x)\right], & p_{2}\left(y_{1}\right)=S_{0}^{1}(x)+\left[S_{0}^{p-1}\left(y_{1}\right)\right]+\frac{1}{S_{0}^{1}(x)}, \\
p_{3}(x)=\left[\lambda_{1} \lambda_{2}-p+1\right] S_{0}^{1}(x), & p_{2}\left(y_{2}\right)=S_{0}^{1}(x)+\left[S_{0}^{1}\left(y_{2}\right)\right]+\frac{1}{S_{0}^{1}(x)},
\end{array}
$$

where $S_{a}^{n}(x)$ and $S_{a}^{n}\left(y_{i}\right)$ are defined as in (11).

Proof Let $p$ be an odd prime. We first need to show that $\mathcal{P}_{E}$ in the theorem actually presents a semi-direct product of a free abelian monoid rank two by a finite cyclic monoid as defined in (2). To do that, we just have to ensure that Equation (1) holds.

From $\mathcal{P}_{E}$, one can easily obtain a diagonal matrix $\mathcal{M}=\left[\begin{array}{cc}p-1 & 0 \\ 0 & 1\end{array}\right]$ which $\operatorname{det} \mathcal{M}=\lambda_{1} \lambda_{2}=p-1$ for eigenvalues $\lambda_{1}$ and $\lambda_{2}$. Moreover, by Lemma 1 , we obviously get $(p-1)^{2 p+1} \equiv p-1$ mod $p$ similarly as the other $2 p+1$ th powers of entries. Thus we obtain

$$
\mathcal{M}^{2 p+1} \equiv \mathcal{M} \quad \bmod p,
$$

which implies that $\mathcal{P}_{E}$ actually presents the required semi-direct product. Hence, as indicated in the proof of Theorem 6 , we have the generating functions $p_{1}(x)$ and $p_{2}\left(y_{i}\right)$ for $1 \leq i \leq 2$ (by Theorem 4 ).

However, again as in the proof of Theorem 6, we need to find a new function (array polynomial) which is related to the minimality (having inefficiency) status of $\mathcal{P}_{E}$. To do that, we will take into account the same way which was obtaining the function $p_{3}(x)=$ $\left[\lambda_{1} \lambda_{2}-1\right] S_{0}^{1}(x)$ in Theorem 6. From Proposition 1, we know that any semi-direct product presentation is not efficient if the determinant of the base matrix $\mathcal{M}$ is equivalent to 0 or $p-1$ by modulo $p$, and also by Proposition 2, this presentation is minimal (whilst it is efficient) if the prime $p$ is odd and the entries of the main diagonal are $p-1$ and 1 (or vice versa) in the diagonal matrix. It is clear that all these situations are suitable for our case in this proof. Therefore, since we have $\lambda_{1} \lambda_{2}=p-1$, there must exist $\left[\lambda_{1} \lambda_{2}-(p-1)\right] S_{0}^{1}(x)$ as a generating function.

Hence the result.

By Theorem 5, one can express Theorem 7 as in the following corollary. In fact, this result is the minimality version of Corollary 1 . We will again omit the proof since it is quite clear by taking $\lambda_{1} \lambda_{2}=p-1$ and $\lambda_{1}+\lambda_{2}=\operatorname{tr}(\mathcal{M})=p$ in (12). 
Corollary 2 Let us consider the minimal but inefficient presentation $\mathcal{P}_{E}$ in Theorem 7. Then it accepts $p(v)=S_{0}^{2}(v)-p S_{0}^{1}(v)+p-1$ as a generating function, where $v$ represents three-ordered variables $\left(x, y_{1}, y_{2}\right)$.

Similarly to Corollary 1 , the function $p(v)$ in the above corollary can be taken as the simplest (reduced) form being in the same congruence class as all other generating functions $p(v)$ that represent this minimal presentation.

Remark 2 From Corollary 2 and by considering Theorem 1, we can easily deduce that the set of generating functions for a minimal but inefficient presentation can be presented as a single element. Therefore, as pointed out in Remark 1 , this shows the importance of minimality (even holding inefficiency) in the meaning of the study of generating functions.

As a result of Theorem 5 and Corollaries 1, 2, we can state that array polynomials obtained in this paper are congruent to each other and so they construct congruence classes. Since these results are based on the characteristic polynomials, the proof of the following main theorem of this paper can be seen quite similar to the proofs of Proposition 3 and Theorem 1.

Theorem 8 Each array polynomial obtained from the presentation $\mathcal{P}_{E}$ in (2) appears to be a congruence class. Moreover, this presentation is represented by a single type of array polynomials depending on this congruence. This single type may contain a unique congruence class of array polynomials in the case of efficiency or minimality status of $\mathcal{P}_{E}$.

\section{Conclusions}

In this part of the paper, we will discuss some problems which can be thought of as conjectures or conclusions and so can be studied in the future projects.

The first project would be the following: In Conjecture 1, by considering a $2 \times 2$-matrix $\mathcal{M}$ with eigenvalues $\lambda_{1}$ and $\lambda_{2}$, we inquired whether the statement $\operatorname{gcd}(\operatorname{tr}(\mathcal{M}), \operatorname{det}(\mathcal{M}))=1$ is enough to get minimality of the presentation. In fact, if one can manage to extend it to a larger situation such as

$$
\operatorname{gcd}\left(\lambda_{1}+\lambda_{2}+\cdots+\lambda_{n}, \lambda_{1} \lambda_{2} \cdots \lambda_{n}\right)=1
$$

for an $n \times n$-matrix $\mathcal{M}$, then this would cover a more general situation.

The equivalence indicated in Conjecture 2 can probably be obtained by the following idea. Let us think about the generating functions defined in Theorem 4 as of the elements of a topological space $\mathcal{T}_{1}$ and, similarly, the generating functions defined in Theorem 5 as of the elements of a topological space $\mathcal{T}_{2}$. Then, by investigating the homotopy equivalence between $\mathcal{T}_{1}$ and $\mathcal{T}_{2}$, we can check the existence of required equivalence.

Another outcome of this paper can be seen in Remarks 1 and 2. It is understood that to study efficient or minimal presentations (and so with the minimal number of generators) implies the minimal number of generating functions. In addition to the material given in the above paragraph, one can also investigate whether the set, say $B$, of this minimal number of generating functions can be a generating set of this type of functions. Mathematically, we can study whether there exists an equality $\langle B\rangle=\bigcup_{i \in I} G_{i}$, where each $G_{i}$ represents the set of generating functions obtained from the related presentation. 


\section{After all the above discussions, as a general case, one may ask whether the equivalence of constants of generating functions implies the equivalence of generating functions directly.}

\section{Competing interests}

The authors declare that they have no competing interests.

\section{Authors' contributions}

All authors completed the paper together. All authors read and approved the final manuscript.

\section{Author details}

${ }^{1}$ Department of Mathematics, Faculty of Science, Selçuk University, Campus, Konya, 42075, Turkey. ${ }^{2}$ Department of Mathematics, Sungkyunkwan University, Suwon, 440-746, Republic of Korea. ${ }^{3}$ Department of Mathematics, Faculty of Art and Science, Akdeniz University, Campus, Antalya, 07058, Turkey. ${ }^{4}$ Department of Mathematics, Faculty of Arts and Science, Uludag University, Gorukle Campus, Bursa, 16059, Turkey.

\section{Acknowledgements}

Dedicated to Professor Hari M Srivastava.

The first, third and fourth authors are partially supported by Research Project Offices of Selcuk (13701071), Uludag (2012-15 and 2012-19) and Akdeniz Universities, respectively. Also the second author is supported by Sungkyunkwan University BK21 Project, BK21 Math Modeling HRD Div. Sungkyunkwan University, Suwon, Republic of Korea.

\section{Received: 2 December 2012 Accepted: 10 February 2013 Published: 28 February 2013}

\section{References}

1. Elsner, C, Shiokawa, I: On algebraic relations for Ramanujan's functions. Ramanujan J. 29, 273-294 (2012)

2. Simsek, Y, Kim, T, Park, DW, Ro, YS, Jang, LJ, Rim, SH: An explicit formula for the multiple Frobenius-Euler numbers and polynomials. JP J. Algebra Number Theory Appl. 4, 519-529 (2004)

3. Srivastava, HM, Coi, J: Series Associated with the Zeta and Related Functions. Kluwer Academic, Dordrecht (2001)

4. Srivastava, HM: Some generalizations and basic (or $q^{-}$) extensions of the Bernoulli, Euler and Genocchi polynomials. Appl. Math. Inf. Sci. 5, 390-444 (2011)

5. Woodcock, CF: Convolutions on the ring of p-adic integers. J. Lond. Math. Soc. 20(2), 101-108 (1979)

6. Ateş, F, Cevik, AS: The $p$-Cockcroft property of central extensions of groups II. Monatshefte Math. 150, 181-191 (2007)

7. Cevik, AS: The $p$-Cockcroft property of the semi-direct products of monoids. Int. J. Algebra Comput. 13(1), 1-16 (2003)

8. Cevik, AS: Minimal but inefficient presentations of the semi-direct products of some monoids. Semigroup Forum 66 , 1-17 (2003)

9. Cevik, AS, Das, KC, Cangul, IN, Maden, AD: Minimality over free monoid presentations. Hacet. J. Math. Stat. (acceptted)

10. Shiratani, K, Yokoyama, S: An application of p-adic convolutions. Mem. Fac. Sci., Kyushu Univ., Ser. A, Math. 36(1), 73-83 (1982)

11. Birkhoff, GD, Lewis, D: Chromatic polynomials. Trans. Am. Math. Soc. 60, 355-451 (1946)

12. Cardoso, DM, Silva, ME, Szymanski, J: A generalization of chromatic polynomial of a graph subdivision. J. Math. Sci. $182(2), 246-254(2012)$

13. Cangul, IN, Cevik, AS, Simsek, Y: A new approach to connect algebra with analysis: relationships and applications between presentations and generating functions. Bound. Value Probl. 2013, 51 (2013). doi:10.1186/1687-2770-2013-51

14. Remmel, J, Riehl, M: Generating functions for permutations which contain a given descent set. Electron. J. Combin. 17(1), R27 (2010)

15. Srivastava, HM: An equivalence theorem on generating functions. Proc. Am. Math. Soc. 52(1), 159-165 (1975)

16. Ivanov, SV: Relation modules and relation bimodules of groups, semigroups and associative algebras. Int. J. Algebra Comput. 1, 89-114 (1991)

17. Clifford, AH, Preston, GB: The Algebraic Theory of Semigroups. Volumes I and II, 2nd edn. Am. Math. Soc., Providence (1964)

18. Cohen, DE: Combinatorial Group Theory: A Topological Approach. London Mathematical Society Student Texts, vol. 14. Cambridge University Press, Cambridge (1989)

19. Cangul, IN, Cevik, AS, Simsek, Y: Analysis approach to finite monoids. Fixed Point Theory Appl. 2013, 15 (2013) doi:10.1186/1687-1812-2013-15

20. Chang, $\mathrm{CH}, \mathrm{Ha}, \mathrm{CW}$ : A multiplication theorem for the Lerch zeta function and explicit representations of the Bernoulli and Euler polynomials. J. Math. Anal. Appl. 315, 758-767 (2006)

21. Simsek, Y: Generating functions for generalized Stirling type numbers, array type polynomials, Eulerian type polynomials and their applications. arXiv:1111.3848v2 [math.NT] 23 Nov 2011

22. Simsek, Y: Interpolation function of generalized $q$-Bernstein type polynomials and their application. In: Curve and Surface. LNCS, vol. 6920, pp. 647-662. Springer, Berlin (2011)

23. Mismar, MJ, Abu-Al-Nadi, DI, Ismail, TH: Pattern synthesis with phase-only control using array polynomial technique. In: 2007 IEEE International Conference on Signal Processing and Communications (ICSPC 2007), Dubai, UAE, 24-27 November 2007 (2007)

doi:10.1186/1687-1812-2013-44

Cite this article as: Cevik et al.: Some array polynomials over special monoid presentations. Fixed Point Theory and Applications 2013 2013:44 